\title{
Translocation of HSP27 into liver cancer cell nucleus may be associated with phosphorylation and O-GIcNAc glycosylation
}

\author{
KUN GUO $^{1}$, LIN GAN $^{1}$, SHU ZHANG $^{2}$, FENG JIE CUI $^{1}$, WANG CUN $^{2}$, YAN LI $^{1}$, \\ NAN XIAO KANG ${ }^{2}$, MEI DONG GAO ${ }^{1}$ and KUN YIN LIU ${ }^{1,2}$ \\ ${ }^{1}$ Liver Cancer Institute, Zhongshan Hospital, ${ }^{2}$ Research Center for Cancer, Institute of \\ Biomedical Science, Fudan University, Shanghai 200032, P.R. China
}

Received December 8, 2011; Accepted January 16, 2012

DOI: 10.3892/or.2012.1844

\begin{abstract}
It has been reported that the dynamic interplay between O-GlcNAcylation and O-phosphorylation is responsible for altering the activity or localization of heat-shock proteins. The aim of this study was to determine whether dynamic interplay between O-GlcNAcylation and O-phosphorylation of HSP27 in hepatocellular cancer (HCC) cells affect its entry into the nucleus. We demonstrate that the entry of HSP27 into the nucleus correlated with its phosphorylation through transfecting HCC cells with plasmids coding for wild-type HSP27 (HSP27-WT), its non-phosphorylatable (HSP27-3A) and pseudophosphorylated (HSP27-3D) mutants, however, not all of the endogenous or exogenous nuclear HSP27 was modified by phosphorylation. We observed that HSP27 was modified with O-GlcNAc glycosylation in HCC cells and report that at conserved Ser residues of HSP27, alternative phosphorylation and O-GlcNAc modification can be predicted by the YinOYang 1.2 method. Furthermore, after P79350 or combined SB203580 and PUGNAc treatment, increased nuclear import of HSP27-WT and HSP27-3D implied that the entry of HSP27 into the nucleus was not only correlated with phosphorylation, but also with O-GlcNAc glycosylation. Collectively, O-GlcNAcylation of HSP27 in HCC cells may be a novel regulatory mode of HSP27 function, particularly for its entry into the nucleus. Crosstalk or interplay between glycosylation and phosphorylation of HSP27 could regulate its subcellular localization and biological functions in liver cancer.
\end{abstract}

\section{Introduction}

Heat shock proteins (HSPs) are evolutionarily conserved molecules and are the products of several distinct gene fami-

Correspondence to: Dr Kun Yin Liu, Liver Cancer Institute, Zhongshan Hospital, Fudan University, Shanghai 200032, P.R. China E-mail: liu.yinkun@zs-hospital.sh.cn

Key words: hepatocellular cancer, post-translational modification, HSP27, nuclear import lies that are required for cell survival during stress $(1,2)$. It has been implicated that members of the HSP family can promote tumor cell proliferation and inhibit cellular death pathways which result in uncontrolled growth $(3,4)$.

In recent years, roles of heat shock protein 27 (HSP27) in human cancer have been intensively discussed, its aberrant expression implies aggressive tumor behavior, increased resistance to chemotherapy and poor prognosis for the patient (5). HSP27 functions as a molecular chaperone and also as an anti-apoptotic protein to prevent apoptotic cell death triggered by a variety of stimuli (6-8). We have previously shown important roles of HSP27 in liver cancer cell motility and apoptosis in vitro $(9,10)$. These activities can occur throughout the cell or are restricted to the cytoplasmic/nuclear cell compartment. It has been noted that HSP27 may perform other important functions in the cell and, especially, in the nucleus (11-13). Moreover, the entry of HSP27 into the nucleus of cells has been shown to correlate strongly with HSP27 post-translation modification $(14,15)$.

In addition, it is well accepted that the glycosylation of $\beta$-O-linked $\mathrm{N}$-acetylglucosamine (O-GlcNAc) at serine and threonine residues on wide variety of nuclear and cytoplasmic proteins is an abundant and dynamic post-translational modification in multicellular eukaryotes (16). In most aspects, O-GlcNAcylation functions more similarly to protein phosphorylation than to 'classical' protein glycosylation (17). It has been shown in site-mapping studies that O-GlcNAcylation and O-phosphorylation at same serine or threonine hydroxyl moiety present dynamic competition on some proteins. This reciprocal occupancy makes these proteins display different activities or stability (18). Thus, dynamic interplay between O-GlcNAcylation and O-phosphorylation is widespread and quite complex, responsible for regulation of signaling, transcription and other cellular processes (19).

It has been found that O-GlcNAc can mediate stress tolerance and protect cells in some ways by altering intercellular heat-shock proteins (HSPs) levels, their activity or localization, stabilizing protein structure, preventing protein-protein aggregation or modulating signal transduction pathways (18). It implies that elevation in O-GlcNAcylation appears to be an important survival mechanism. Therefore, elucidating dynamic 
post-translational modification of heat shock proteins should improve our understanding of how the HSPs help cells to survive stressful conditions.

In this study, we demonstrated that HSP27 phosphorylation plays a critical role in nuclear translocation and described potential glycosylation and interplay sites of phosphorylation and glycosylation in various domains of HSP27, using the different methods available. Moreover, the entrance of HSP27 into nucleus was affected by not only phosphorylation, but also modification of O-GlcNAcylation.

\section{Materials and methods}

Preparation of cell lysates and nuclear extract. HCC cells (MHCC97L, MHCC97H, HCCLM6) were densely seeded into T-25 flasks and allowed to grow for $24 \mathrm{~h}$ to $80-85 \%$ confluence in high glucose DMEM plus 10\% FBS. Cells were harvested by trypsinization and washed with cold PBS. Cytosolic and nuclear extracts were prepared using nuclear extraction kit (Active Motif, CA, USA) following manufacturer's instructions with addition of phosphatase inhibitor cocktails (Sigma, MO, USA) or O-GlcNAcase inhibitor-PUGNAc (Tocris, MO, USA) wash of the nuclear pellet prior to nuclear lysis. Protein samples were performed a 1:50 dilution with complete lysis buffer and were then determined using BCA Protein Assay.

Immunofluorescence microscopy. HCC cells were plated on glass coverslips and transfected with wild-type or mutant HSP27 expression vectors using Lipofectamine 2000 (Invitrogen, NY, USA) reagent according to the manufacturer's protocol. After SB203580 or PUGNAc treatment, endogenous or exogenous HSP27 were, respectively, detected by HSP27 (Cell Signaling Technology, MA, USA) or Flag mAb (DYKDDDDK Tag antibody) (BioLegend, CA, USA) and Alexa Fluro ${ }^{\circledR 5} 55$ conjugated-secondary antibody (Cell Signaling Technology), cell imaging was performed. Fluorescence images were captured on a confocal microscope (Leica TCS-NT, Mannheim, Germany).

Plasmids and transfection. Plasmids for expression of Flagtagged (DYKDDDDK Tag) human HSP27 mutants with replacement of serines 15, 78 and 82 with alanine (HSP27-3A) and aspartic acid (HSP27-3D) were created by excising a 1014 bp BamHI-HindIII fragment from pENTR/D-TOPOHSP27(3A) and pENTR/D-TOPO-HSP27(3D) and inserting this fragment into the BamHI-HindIII sites of pcDNA3.1-flag.

HCC cells were cultured in $25-\mathrm{cm}^{2}$ flasks in DMEM with $10 \%$ fetal bovine serum (Invitrogen) at $37^{\circ} \mathrm{C}$ and $5 \%$ $\mathrm{CO}_{2}$ atmosphere in humidified incubator until the $80-85 \%$ confluency was reached. For transfection, cells were seeded on 6 -well plates at a density of $1 \times 10^{5}$ cells/well and incubated at $37^{\circ} \mathrm{C}$ and $5 \% \mathrm{CO}_{2}$ atmosphere $24 \mathrm{~h}$ prior to transfection. The transfection mixtures were pipetted dropwise to the cell cultures with $1 \mathrm{ml}$ of freshly added serum-free medium and allowed to incubate for $6 \mathrm{~h}$ at $37^{\circ} \mathrm{C}$ and $5 \% \mathrm{CO}_{2}$. The final concentration of plasmid DNA was $2 \mu \mathrm{g}$ per well. Six hours after transfection, medium was replaced with $1 \mathrm{ml}$ of fresh growth medium (containing serum) and incubated at $37^{\circ} \mathrm{C}$. The post-transfection incubation time was $48 \mathrm{~h}$ for western blotting of flag tag.
Western blot analysis. Equal amount of protein samples were run on a $10 \%$ SDS polyacrylamide gel and then transferred onto a polyvinylidene fluoride membrane (Millipore Corp., Bedford, MA, USA). Membranes were blocked for $1 \mathrm{~h}$ at room temperature in TBS buffer with $0.05 \%$ Tween-20 containing $5 \%$ non-fat milk or $5 \% \mathrm{BSA}$ and incubated overnight at $4^{\circ} \mathrm{C}$ with HSP27 or phosphor-HSP27 antibody (dilution, 1:1000; Cell Signaling Technology) or O-GlcNAc antibody (dilution, 1:1000; Santa Cruz Biotechnology, CA, USA) or anti-Flag (dilution, 1:1000; Santa Cruz Biotechnology) diluted in TBST buffer. After washing, membrane was incubated for $1 \mathrm{~h}$ at room temperature with goat anti-rabbit or rabbit anti-mouse IgG conjugated with horseradish peroxidase (Promega, Madison, WI, USA). After rinsing, subsequent detection was performed using the ECL western blot system (SuperSignal West Pico Chemiluminescent Substrate, Pierce, Rockford, IL, USA) according to the manufacturer's instruction.

ELISA. To detect and compare HSP27 level and phosphor-HSP27 level, a solid sandwich enzyme-linked immunosorbent assay (ELISA, Cell Signaling Technology) was carried out according to the manufacturer's manual. For endogenous HSP27, 96-well plates were coated with capture antibody-HSP27 mouse mAb (Cell Signaling Technology). For exogenous HSP27, $100 \mu \mathrm{l}$ of anti-flag antibody $(20 \mu \mathrm{g} / \mathrm{ml})$ in coating buffer [0.1 M sodium carbonate ( $\mathrm{pH} 9.6)$ with $0.02 \%$ sodium azide] was added to each well in 96-well pro-coated plates and incubated for $24 \mathrm{~h}$ at room temperature in a humid chamber. After blocking with $0.1 \%$ BSA in PBS buffer, it was kept for the next assay. All incubation steps were then separated by three washing steps. Diluted nuclear lysate $(100 \mu \mathrm{l})$ was added to the microwell and incubated overnight at $4^{\circ} \mathrm{C}$. Detection antibody was added to each well and then incubated for $1 \mathrm{~h}$ at $37^{\circ} \mathrm{C}$. After washing, HRP-linked secondary antibody was added and incubated for $30 \mathrm{~min}$ at $37^{\circ} \mathrm{C}$. After washing, TMB substrate was added to each well and incubated for $10 \mathrm{~min}$ at $37^{\circ} \mathrm{C}$. Stop solution was added to each well and shaken for a few seconds. Absorbance was read at $450 \mathrm{~nm}$ within $30 \mathrm{~min}$ after adding stop solution. All samples were tested in duplicate and a negative control format was established in order to detect non-specific signals.

Purification of nuclear HSP27 from HCC cells. Equal amounts of total nuclear protein of each HCC cell line (1 mg) were applied to a HiTrap NHS-activated HP (GE Healthcare, Uppsala, Sweden) column that was coupled with $500 \mu \mathrm{l}$ of anti-human HSP27 or anti-Flag antibody (Santa Cruz Biotechnology). The endogenous or exogenous HSP27 bound to the column was eluted and then concentrated by ultafiltration device (Pall Corp., Port Washington, NY). The purified endogenous or exogenous HSP27 was subjected to SDS-PAGE and used for phosphorylation and O-GlcNAcylation analysis.

PTM prediction methods. Methods used for predicting potential N-linked or O-linked glycosylation sites include NetNGlyc 1.0 (http://www.cbs.dtu.dk/services/NetNGlyc/) NetOGlyc 3.1 (http://www.cbs.dtu.dk/services/NetOGlyc/) which predict $\mathrm{O}$-glycosylation sites in HSP27 proteins. To predict potential of O-GlcNAc modification and Yin Yang sites in human HSP27, DictyOGlyc 1.1 (http://www.cbs.dtu. $\mathrm{dk} /$ services/DictyOGlyc/) and YinOYang 1.2 (http://www.cbs. 

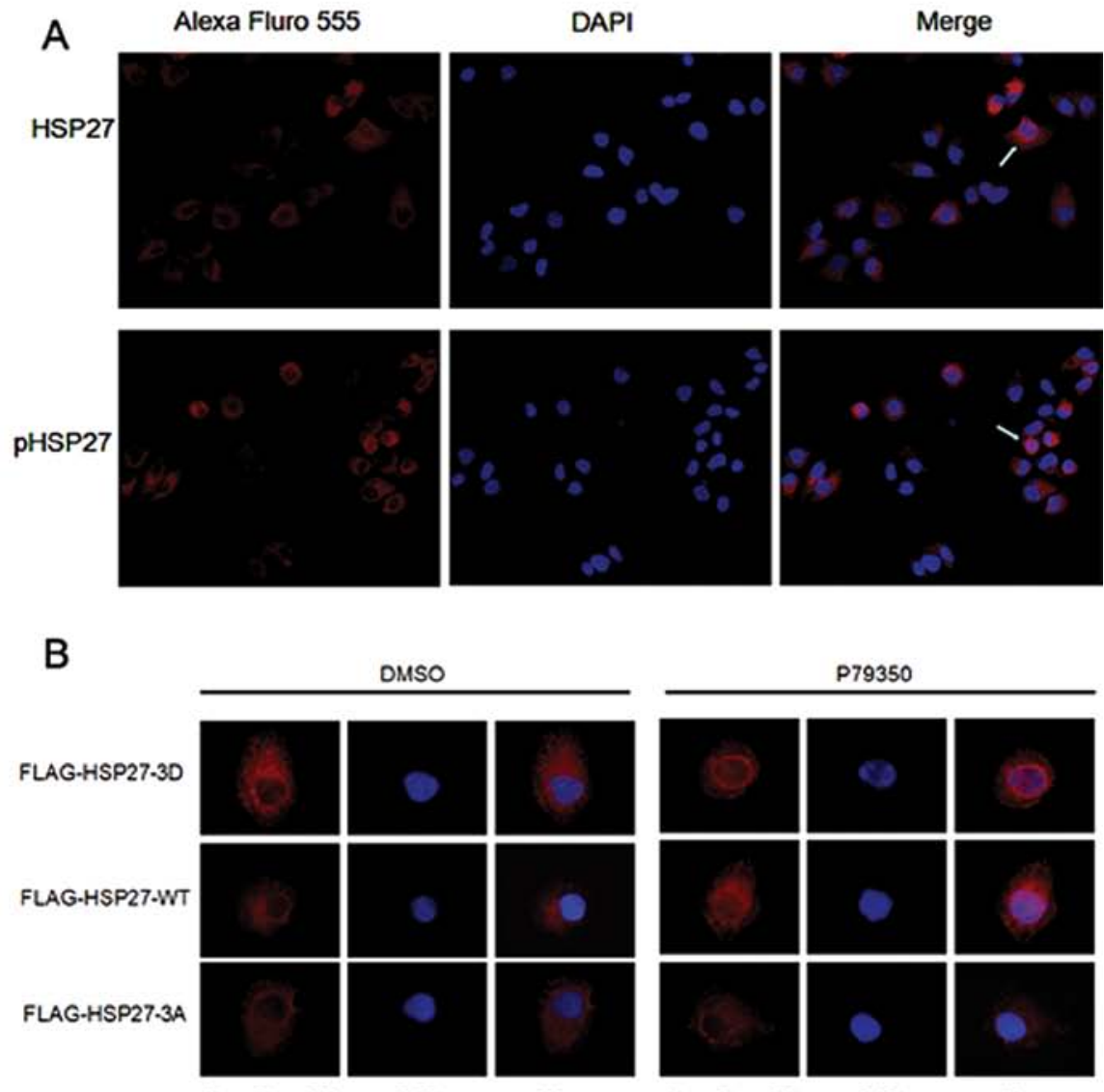

DMSO

P79350

C

Alexa Fluro 585
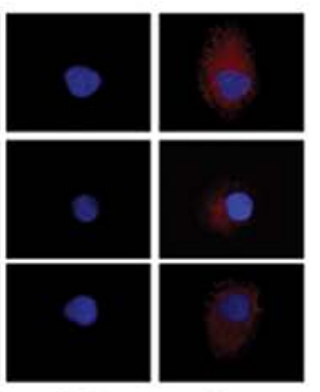

DAPI

Merge
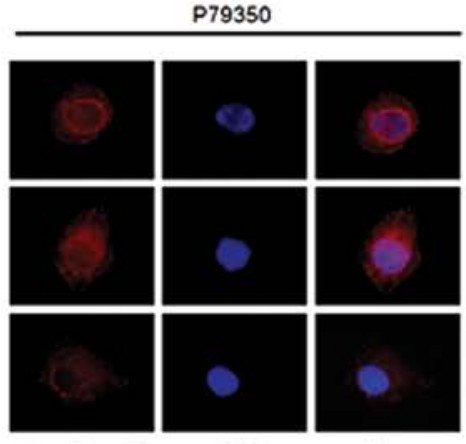

Nexa Fluro 555

DAPI

Merge
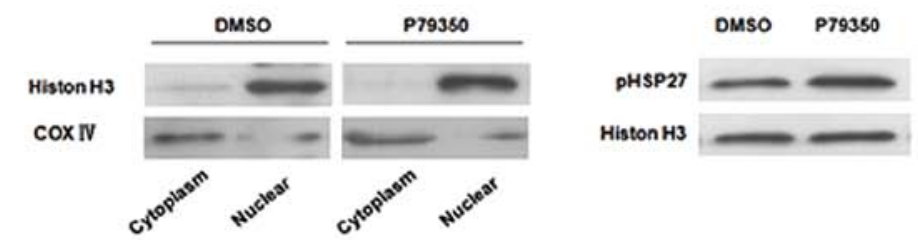

D

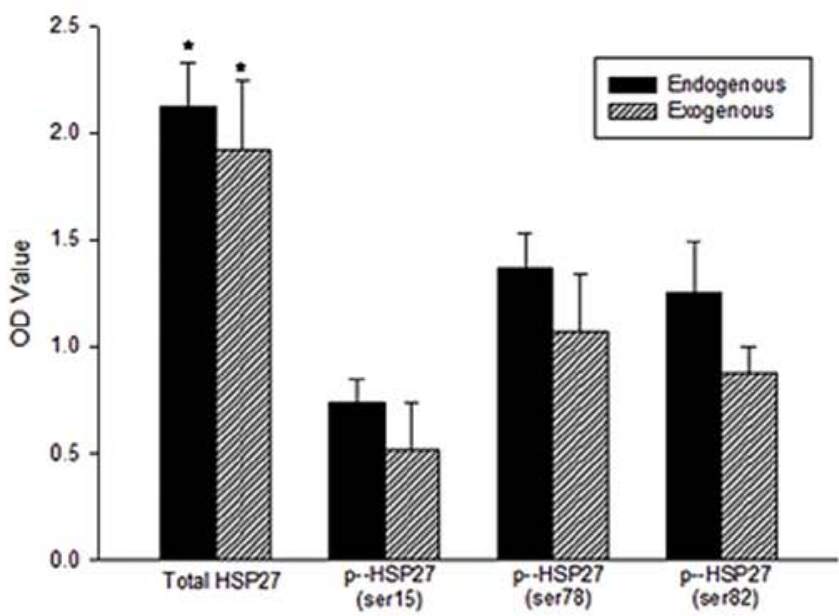

Figure 1. The entry of HSP27 into nucleus of HCC cells correlated with phosphorylation. (A) MHCC97H cells were washed with PBS, fixed and incubated for $1 \mathrm{~h}$ with primary antibodies mouse monoclonal anti-HSP27 and anti-phospho(s82) HSP27 and then probed for $1 \mathrm{~h}$ with Alexa Fluro ${ }^{\circledR 555}$ (red) conjugatedsecondary antibody. All nuclei were stained with DAPI (blue). These confocal immunofluorescence images were merged to show localization of HSP27 and phosphor-HSP27(s82), especial in the nucleus. Localization of HSP27 and phosphor-HSP27 in MHCC97L and HCCLM6 was similar to that in MHCC97H (data not shown). (B) MHCC97H cells were transfected with pcDNA3.1-FLAG-HSP27, pcDNA3.1-FLAG-HSP27-3A and pcDNA3.1- FLAG-HSP27-3D and confocal immunofluorescence images were acquired as (A) described. (C) Nuclear proteins of MHCC97H cells with P79350 treatment were extracted and the extract efficiency was confirmed by nuclear marker-Histone H3 and mitochondrial marker-COX IV (left), then levels of phosphorylated(S82) HSP27 in nucleus was determined by western blot analysis (right). (D) In P79350 treatment condition, levels of total or phosphorylated (ser15/ser78/ser82) endogenous or exogenous HSP27 (FLAG-HSP27-WT) in nuclear extract were detected by ELISA kit and OD450 readings are shown. The results represent means of triplicates; "statistically different from phosphorylation at $\mathrm{p}<0.05$. 


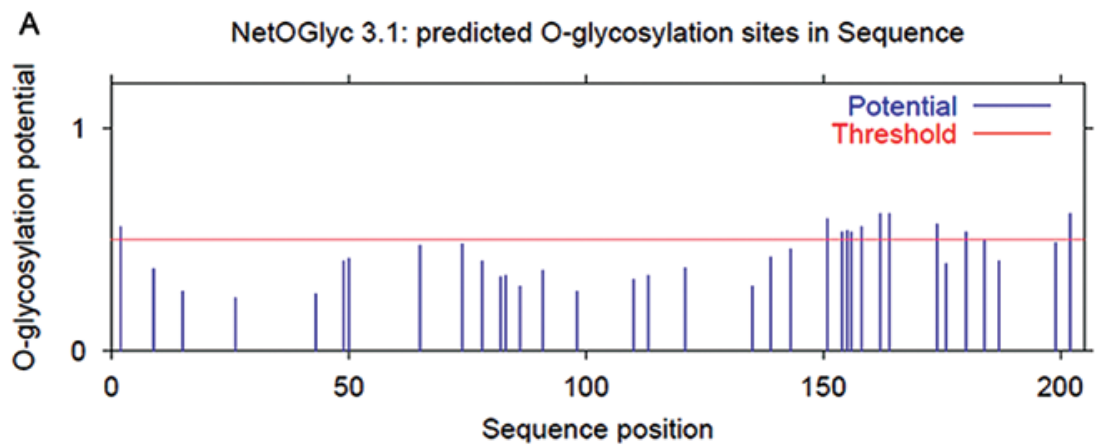

B

YinOYang 1.2: predicted O- $\beta$-GIcNAc sites in Sequence

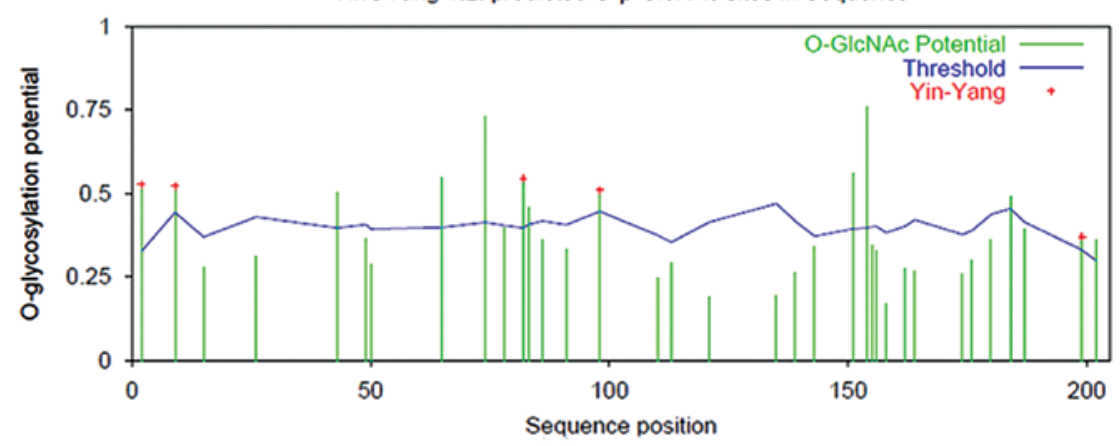

C

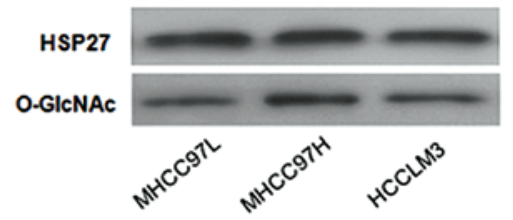

Figure 2. Glycosylation of HSP27 protein in HCC cell lines was predicted online and re-confirmed by O-GlcNAc antibody. (A) Graphic representation of potential for O-Glycosylation modification in the human HSP27 sequence. (B) Graphic representation of potential for O-GlcNAc modification in serine and threonine residues in the human HSP27 sequence. Green vertical lines show the potential of S/T residues for O-GlcNAc modification and light blue horizontal wavy line shows threshold for modification potential. The Yin Yang sites with red asterisk at the top. (C) Purified HSP27 protein from MHCC97L, MHCC97H and HCCLM6 was detected by western blot analysis with specific anti-O-GlcNAc antibody (CTD110.6).

dtu.dk/services/YinOYang/) were used. The method has the capability to predict the sites known as Yin Yang sites that can be O-GlcNAc modified and alternatively phosphorylated. The threshold value used by YinOYang 1.2 varies depending upon surface accessibility of the different amino acid residues.

Statistical analysis. Protein bands, developed by multiple exposure of X-ray film to assure exposure in the linear range, were scanned and quantified using the Quantity One software program. Mean, SD, SE and Student's t-tests were calculated using SPSS 13.0 program. These data were then grouped and analyzed statistically as shown. For the paired t, this is stated; if unpaired, it is referred to simply as Student's t-test.

\section{Results}

The entry of HSP27 into nucleus of HCC cells correlates with phosphorylation. It was reported that a striking feature of HSP27 is its stress inducible entry into nuclei of some cells (20). To corroborate this suggestion, we observed endogenous total and phosphorylated HSP27 in HCC cell nucleus. It was found that although endogenous HSP27 was predominantly located in the cytoplasm of HCC cells without any treatment, non-phosphorylated and phosphorylated HSP27 also localized in nuclear speckles and distributed with intranuclear granules in interphase cells (Fig. 1A). Moreover, we transfected HCC cells with plasmids coding for wild-type HSP27 (FLAG-HSP27-WT) and its non-phosphorylatable ( $\rightarrow$ A) (FLAG-HSP27-3A) and pseudophosphorylated ( $\rightarrow$ D) (FLAG-HSP27-3D) mutants. Under control condition, exogenous HSP27-WT and HSP27-3A were primarily present in the cytoplasm, while a small proportion of HSP27-3D showed nuclear localization (Fig. 1B). Upon P79350 (50 nM), an activator of p38MAPK, FLAG-HSP27-3D which conglomerated around or in the nucleus conspicuously increased and FLAG-HSP27-WT which primarily accumulated in the nucleus also increased, whereas FLAG-HSP27-3A slighly or not at all (Fig. 1B). In addition, the nuclear accumulation was reconfirmed by western blot analysis, which showed nuclear fraction was successfully extracted from HCC cells and an increased amount of endogenous phosphorylated HSP27 in the nuclear fraction of non-transfected HCC cells upon P79350 treatment (Fig. 1C). These changes of localization suggested that nuclear import of HSP27 may be relevant to phosphorylation.

However, it was also found that, in P79350-treatment condition, endogenous or exogenous HSP27 (FLAG-HSP27-WT) which accumulated in the nucleus of HCC cells was not totally 

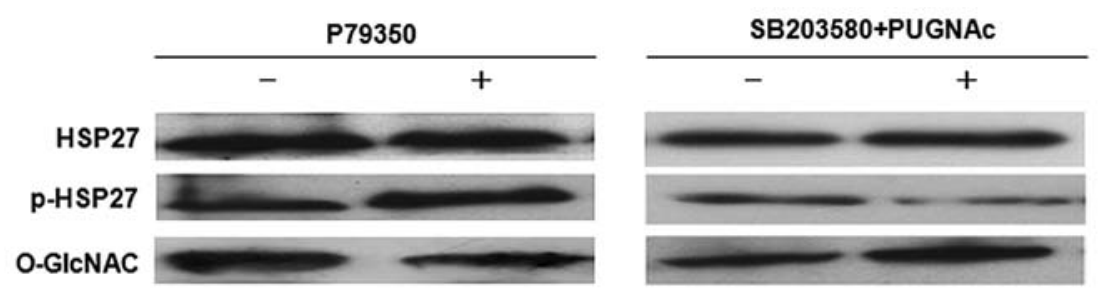

Figure 3. HSP27 in HCC cell lines are modified by interplay of phosphorylation and O-GlcNAc modification. MHCC97H cells were treated with P79350 $(50 \mathrm{nM})$ or SB203580 $(5 \mu \mathrm{M})$ and PUGNAc $(100 \mu \mathrm{M})$, respectively. Purified HSP27 proteins by HiTrap NHS-activated HP were immunoblotted with antiphospho(s82) HSP27 and anti-O-GlcNAc antibody (CTD110.6). HSP27 serves as a loading control.

A
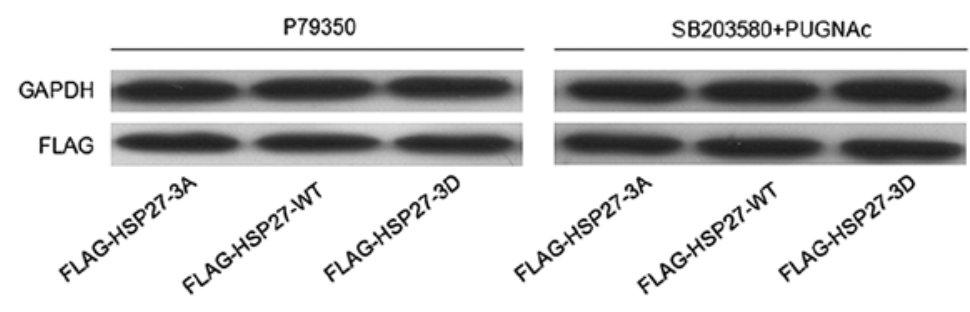

B

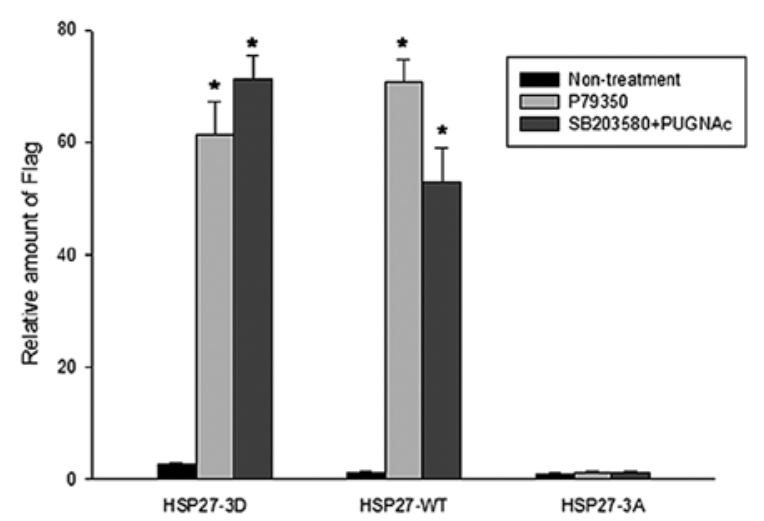

Figure 4. Phosphorylation and O-GlcNAc glycosylation may be responsible for nuclear import of HSP27. (A) Under P79350 or SB203580 and PUGNAc stimulation, MHCC97H cells were transfected with plasmids coding for FLAG-HSP27-3A, FLAG-HSP27-WT and FLAG-HSP27-3D, then levels of Flag in whole cell extract were evaluated by anti-Flag antibody. (B) Levels of Flag in same amount of nuclear extract from these transfected cells were evaluated by immunoblotting with Flag antibody (left). Densitometry of bands from nuclear flag western blot analysis is shown (right). The results represent means of triplicates; "statistically different from non-treatment at $\mathrm{p}<0.05$.

phosphorylated HSP27, because total HSP27 was more than phosphorylated HSP27, as shown in ELISA assay that OD values of endogenous nuclear total HSP27 and phosphor-HSP27 (ser15), phosphor-HSP27(ser78), phosphor-HSP27(ser82) from non-transfected HCC cells was, respectively, $2.12 \pm 0.21$, $0.74 \pm 0.11,1.37 \pm 0.16$ and $1.25 \pm 0.24$; those of Flag purified total HSP27 and phosphor-HSP27 (ser15/ser78/ser82) from transfected HCC cells was, respectively, $1.92 \pm 0.32,0.52 \pm 0.22$, $1.07 \pm 0.27$ and $0.88 \pm 0.12$ (Fig. 1D). It indicated there may be other modification forms of HSP27 in HCC cells involved in transportation into the nucleus.

Prediction of HSP27 glycosylated sites and O-GlcNAc antibody confirmation. Prediction results of HSP27 for different O-linked and N-linked glycosylation sites showed that the protein had the potential for O-linked glycosylation, but not for N-linked glycosylation (Fig. 2A). Among O-linked glycosylation sites, O-GlcNAc was the most frequent. The prediction results for O-GlcNAc modification by YinOYang 1.2 showed that there were 13 potential sites highly predicted to be modified by O-GlcNAc, 9 on Ser residues, at positions 9, 43, 65, $74,82,83,98,154$ and 199 and 4 on Thr residues at positions 2, 151, 184 and 202 (Fig. 2B). O-GlcNAc modification of HSP27 in HCC cells (MHCC97L, MHCC97H, HCCLM6) was reconfirmed by western blot analysis with O-GlcNAc antibody (CTD110.6) (Fig. 2C). Moreover, it was shown that all the possible potential Yin Yang sites which were predicted by the YinOYang 1.2 method but among these only five are most likely to be Yin Yang sites (Fig. 2B).

The nucleus import of HSP27 in HCC cells correlates with not only phosphorylation, but also its $O$-GlcNAc glycosylation. In this study, HCC cells were treated with P79350 or combination of SB203580 (an inhibitor of p38MAPK) and PUGNAc (an inhibitor of O-GlcNAcase) to make intercellular HSP27 phosphorylated or O-GlcNAcylated status. Dynamic changes 
in GlcNAcylation and phosphorylation were first observed by immunoblotting with antibodies against O-GlcNAc and phosphorylated HSP27. To ensure that stress responses, which might obviously trigger unexpected changes of phosphorylation, would not initiate early, $0.5 \mathrm{~h}$ of PUGNAc $(100 \mu \mathrm{M})$ and SB203580 $(5 \mu \mathrm{M})$ treatment was chosen. However, a $0.5-\mathrm{h}$ time course is long enough for P79350 (50 nM) to elevate phosphorylation of HSP27. As shown in Fig. 3, $0.5 \mathrm{~h}$ of treatment by PUGNAc and SB203580 induced significantly elevated O-GlcNAcylation levels of HSP27, whereas P79350 significantly increased phosphorylation.

To further observe the roles of phosphorylation and O-GlcNAc glycosylation for nuclear import of HSP27, HCC cells were transfected with plasmids coding for FLAGHSP27-3A and FLAG-HSP27-3D, then followed by P79350, or SB203580 and PUGNAc treatment. After 48 h, whole cell extract from these transfectants were evaluated by immunoblotting with Flag antibody. It showed that flag levels among these post-transfected cells were similar, suggesting whole exogenous HSP27 levels were similar (Fig. 4A). Thus, levels of Flag in nuclear extract represent the amount of nuclear exogenous HSP27. As Fig. 4B shows, P79350 treatment increased nuclear import of FLAG-HSP27-WT and FLAG-HSP27-3D, but FLAG-HSP27-3A not at all or slightly, compared with increasing of FLAG-HSP27-WT and FLAG-HSP27-3D; whereas combined SB203580 $(5 \mu \mathrm{M})$ and PUGNAc $(100 \mu \mathrm{M})$ treatment also led to accumulation of FLAG-HSP27-WT and FLAG-HSP27-3D in the nucleus, increasing extent of FLAGHSP27-WT was higher. This result implied that the entry of HSP27 into the nucleus of HCC cells was correlated with phosphorylation, however, phosphorylation is necessary but not sufficient, its O-GlcNAc glycosylation was also involved.

\section{Discussion}

HSP27 is a ubiquitous and abundantly expressed member of the small Hsp family which has many diverse functions including chaperone activity, mRNA stabilization, inhibition of apoptosis, and modulation of actin polymerization (21). The functional activity of HSP27 is prominently determined by phosphorylation state of serine residues at positions 15,78 and 82 , which is activated by some intercellular kinases $(22,23)$. It has been verified that alteration of HSP27 phosphorylation state at some important sites is often associated with relocalization of HSP27 from the cytoplasm into the nucleus or perinuclear area (24). In this study, it was found upon P79350 treatment, that some endogenous phosphorylated HSP27 and exogenous pseudophosphorylated FLAG-HSP27-3D and FLAG-HSP27-WT conspicuously conglomerated in the nucleus of HCC cells; whereas FLAG-HSP27-3A slightly or not at all. The result suggested HSP27 displayed an intermediate ability to enter nuclei of HCC cells upon its phosphorylation level increasing. In addition, FLAG-HSP27-3D showed spontaneously accumulated ability in the nucleus without any treatment, although not all FLAG-HSP27-3D. It also implied the phosphorylation, but not stress itself, mediated entry of HSP27 into the nucleus of HCC cells. Interestingly, biochemical isolates of nuclear HSP27 contain also non-phosphorylated HSP27. It suggested that in addition to phosphorylation, there is another mechanism determining efficient entry of HSP27 into the nucleus. Thus, the regulatory mechanism of HSP27 entry into nucleus needs to be elucidated further.

O-GlcNAc is one of many post-translational modifications that contribute to the complexity of the cell's phenotype, attributable to multiple, competing, and various combinations of site occupancy $(25,26)$. Modification of O-GlcNAcylation has been detected on a wide variety of proteins, including RNA polymerase II, transcription factors, kinases, phosphatases, cytoskeletal proteins, nuclear hormone receptors, nuclear pore proteins, signal transduction molecules and actin regulatory proteins $(18,19)$.

Many of the above proteins are dynamically modified by O-GlcNAc or O-phosphate at the same or adjacent sites. The reciprocal occupancy can modulate protein functions and affect cell behavior $(18,21)$. On the contrary, abnormal crosstalk between GlcNAcylation and phosphorylation can result in cancer, diabetes and neurodegenerative disease.

In this study, glycosylation modification of HSP27 was predicted by online software, since computer-assisted methods are useful in assessing the modification potential of a given protein (27). Moreover, glycosylation and O-GlcNAc modification of HSP27 was also identified by anti-O-GlcNAc antibody, suggesting the existence of O-GlcNAc modification on HSP27 protein, consistent with some literature (28).

As expected, p38MAPK activator-P79350 treatment led to increased phosphorylation on HSP27, whereas O-GlcNAcylation decreased. In contrast, combined SB203580 and PUGNAc treatment induced decreased phosphorylation of HSP27 and increased its O-GlcNAcylation. It indicated that changes in phosphorylation induced by manipulating kinase activities were correlated with reciprocal dynamics of O-GlcNAcylation on HSP27 protein. In addition, upregulated phosphorylation and O-GlcNAcylation of HSP27 promoted its accumulation in nucleus, whereas negative mutant FLAGHSP27-3A not at all or slightly. These results implied that in HCC cells, except phosphorylation, O-GlcNAcylation also promoted the entry of HSP27 into the nucleus.

Our study does not address the issue of how reciprocal dynamics between O-GlcNAcylation and phosphorylation affect the entry of HSP27 into the nucleus. For example, whether phosphorylation at all of these sites is required in the process of HSP27 entry into nucleus, and which mechanism cross-talk or interplay between O-GlcNAcylation and phosphorylation and arise from this, is not yet clear. However, previous and the current data are sufficient to demonstrate that entry of human HSP27 into the nucleus indeed requires phosphorylation and O-GlcNAcylation. In addition, although phosphorylation mimicking mutant FLAG-HSP27-3D is absent of free hydroxyls, its nuclear import increased upon p38MAPK activation. One possibility is that p38MAPK activation provides a kind of stress condition which mediated FLAG-HSP27-3D mutant recruitment of nuclear speckles for refolding the unfolded nucleocytoplasmic shuttle proteins, because FLAG-HSP27-3D presents the best refolding rates (29).

In summary, glycosylation modification of specific protein is a rapidly growing field. O-GlcNAcylation of HSP27 in HCC cells, which was identified in the study, may be a novel regulatory state of HSP27 function, especially for the entry of HSP27 into nucleus of HCC cells. Crosstalk or interplay 
between glycosylation and phosphorylation of HSP27 in HCC cells could regulate its subcellular localization, association with binding partners and activity in response to cellular signals or stages. Nevertheless, further studies are required to determine this mechanism.

\section{Acknowledgements}

We thank sincerely Dr R. Gerthoffer and Glen Douglas, University of South Alabama for providing pENTR/D-TOPOHSP27(3A) and pENTR/D-TOPO-HSP27(3D). This study was supported by the following grants: China National HighTech Research Development Program (2006AA02A308), China National Key Projects for Infectious Disease (2008ZX 10002-021 and 2008ZX10002-017), National Natural Scientific Funding (81001057), Fudan Young Scientist Foundation (08FQ34) and National New Lecturer Foundation (200802461034).

\section{References}

1. Sitia R and Molteni SN: Stress, protein (mis)folding, and signaling: the redox connection. Sci STKE 2004: pe27, 2004

2. Isaacs JS, Xu WP and Neckers L: Heat shock protein 90 as a molecular target for cancer therapeutics. Cancer Cell 3: 213-217, 2003.

3. Gimenez Ortiz A and Montalar Salcedo J: Heat shock proteins as targets in oncology. Clin Transl Oncol 12: 166-173, 2010.

4. Calderwood SK, Khaleque MA, Sawyer DB and Ciocca DR: Heat shock proteins in cancer: chaperones of tumorigenesis. Trends Biochem Sci 31: 164-172, 2006.

5. Arrigo AP, Simon S, Gibert B, Kretz-Remy C, Nivon M, Czekalla A, Guillet D, Moulin M, Diaz-Latoud C and Vicart P: Hsp27 (HspB1) and $\alpha B$-crystallin (HspB5) as therapeutic targets. FEBS Lett 581: 3665-3674, 2007.

6. Garrido C, Brunet M, Didelot C, Zermati Y, Schmitt E and Kroemer G: Heat shock proteins 27 and 70: anti-apoptotic proteins with tumorigenic properties. Cell Cycle 5: 2592-2601, 2006.

7. Concannon CG, Gorman AM and Samali A: On the role of Hsp27 in regulating apoptosis. Apoptosis 8: 61-70, 2003.

8. Hassan S, Biswas MH, Zhang C, Du C and Balaji KC: Heat shock protein 27 mediates repression of androgen receptor function by protein kinase D1 in prostate cancer cells. Oncogene 284: 386-4396, 2009.

9. Guo K, Kang NX, Li Y, Sun L, Gan L, Cui FJ, Gao MD and Liu KY: Regulation of HSP27 on NF-kB pathway activation may be involve $\mathrm{d}$ in metastatic hepatocellular carcinoma cells apoptosis. BMC Cancer 9: 100, 2009.

10. Guo K, Liu Y, Zhou H, Dai Z, Zhang J, Sun R, Chen J, Sun Q, $\mathrm{Lu}$ W, Kang X and Chen P: Involvement of protein kinase C $\beta$-extracellular signal-regulating kinase ${ }_{1 / 2} / \mathrm{p} 38$ mitogen-activated protein kinase-heat shock protein 27 activation in hepatocellular carcinoma cell motility and invasion. Cancer Sci 99: 486-496, 2008.

11. Bryantsev AL, Chechenova MB and Shelden EA: Recruitment of phosphorylated small heat shock protein Hsp27 to nuclear speckles without stress. Exp Cell Res 313: 195-209, 2007.
12. Michaud S, Lavoie S, Guimond MO and Tanguay RM: The nuclear localization of Drosophila Hsp27 is dependent on a monopartite arginine-rich NLS and is uncoupled from its association to nuclear speckles. Biochim Biophys Acta 1783: 1200-1210, 2008.

13. Zhang D, Wong LL and Koay ES: Phosphorylation of Ser78 of Hsp27 correlated with HER-2/neu status and lymph node positivity in breast cancer. Mol Cancer 6: 52, 2007.

14. Den Engelsman J, Gerrits D, de Jong WW, Robbins J, Kato K and Boelens WC: Nuclear import of $\alpha \mathrm{B}$-crystallin is phosphorylation-dependent and hampered by hyperphosphorylation of the myopathy-related mutant R120G. J Biol Chem 280: 37139-37148, 2005.

15. Geum D, Son GH and Kim K: Phosphorylation-dependent cellular localization and thermoprotective role of heat shock protein 25 in hippocampal progenitor cells. J Biol Chem 277: 19913-19921, 2002.

16. Hart GW, Housley MP and Slawson C: Cycling of O-linked $\beta-N$-acetylglucosamine on nucleocytoplasmic proteins. Nature 446: 1017-1022, 2007.

17. Slawson C and Hart GW: Dynamic interplay between O-GlcNAc and O-phosphate: the sweet side of protein regulation. Curr Opin Struct Biol 13: 631-636, 2003.

18. Wells L, Vosseller K and Hart GW: Glycosylation of nucleocytoplasmic proteins: signal transduction and O-GlcNAc. Science 291: 2376-2378, 2001.

19. Zachara NE and Hart GW: The emerging significance of O-GlcNAc in cellular regulation. Chem Rev 102: 431-438, 2002.

20. Adhikari AS, Sridhar Rao K, Rangaraj N, Parnaik VK and Mohan Rao C: Heat stress-induced localization of small heat shock proteins in mouse myoblasts: intranuclear lamin $\mathrm{A} / \mathrm{C}$ speckles as target for $\alpha \mathrm{B}$-crystallin and Hsp25. Exp Cell Res 299: 393-403, 2004.

21. Lasa M, Mahtani KR, Finch A, Brewer G, Saklatvala J and Clark AR: Regulation of cyclooxygenase 2 mRNA stability by the mitogen-activated protein kinase p38 signaling cascade. Mol Cell Biol 204: 265-4274, 2000

22. Hedges JC, Dechert MA, Yamboliev IA, Martin JL, Hickey E, Weber LA and Gerthoffer WT: A role for p38(MAPK)/HSP27 pathway in smooth muscle cell migration. J Biol Chem 274: 24211-24219, 1999.

23. Benndorf R, Engel K and Gaestel M: Analysis of small Hsp phosphorylation. Methods Mol Biol 99: 431-445, 2000.

24. McClaren M and Isseroff RR: Dynamic changes in intracellular localization and isoforms of the $27-\mathrm{kD}$ stress protein in human keratinocytes. J Invest Dermatol 102: 375-381, 1994.

25. Vosseller K, Wells L and Hart GW: Nucleocytoplasmic O-glycosylation: O-GlcNAc and functional proteomics. Biochimie 83: 575-581, 2001.

26. Kouzarides T: Acetylation: a regulatory modification to rival phosphorylation. EMBO J 19: 1176-1179, 2000.

27. Nielsen H, Brunak S and von Heijne G: Machine learning approaches for the prediction of signal peptides and other protein sorting signals. Protein Eng 12: 3-9, 1999.

28. Love DC and Hanover JA: The hexosamine signaling pathway: deciphering the 'O-GlcNAc code'. Sci STKE 2005: re13, 2005.

29. Bryantsev AL, Kurchashova SY, Golyshev SA, Polyakov VY, Wunderink HF, Kanon B, Budagova KR, Kabakov AE and Kampinga HH: Regulation of stress-induced intracellular sorting and chaperone function of Hsp27 (HspB1) in mammalian cells. Biochem J 407: 407-417, 2007. 